\title{
Bur open Integrated oncogeriatric approach: a systematic review of the literature using concept analysis
}

\author{
Dominique Tremblay, ${ }^{1}$ Kathleen Charlebois, ${ }^{2}$ Catherine Terret, ${ }^{3}$ Sonia Joannette, ${ }^{4}$ \\ Jean Latreille ${ }^{5}$
}

To cite: Tremblay $D$, Charlebois K, Terret C, et al. Integrated oncogeriatric approach: a systematic review of the literature using concept analysis. BMJ Open 2012;2:e001483.

doi:10.1136/bmjopen-2012001483

- Prepublication history and additional material for this paper are available online. To view these files please visit the journal online (http://dx.doi.org/10.1136/ bmjopen-2012-001483).

Received 22 May 2012 Accepted 12 November 2012

This final article is available for use under the terms of the Creative Commons Attribution Non-Commercial 2.0 Licence; see http://bmjopen.bmj.com

For numbered affiliations see end of article

Correspondence to Dr Dominique Tremblay; dominique.tremblay2@ usherbrooke.ca

\section{ABSTRACT}

Objectives: The purpose of this study was to provide a more precise definition of an integrated oncogeriatric approach (IOGA) through concept analysis.

Data sources: The literature was reviewed from January 2005 to April 2011 integrating three broad terms: geriatric oncology, multidisciplinarity and integrated care delivery models.

Study eligibility criteria: Citation selection was based on: (1) elderly cancer patients as the study population; (2) disease management and (3) case studies, intervention studies, assessments, evaluations and studies. Inclusion and exclusion criteria were refined in the course of the literature search.

Interventions: Initiatives in geriatric oncology that relate to oncology services, social support services and primary care services for elderly cancer patients.

Participants: Elderly cancer patients aged 70 years old or more.

Study appraisal and synthesis methods: Rodgers' concept analysis method was used for this study. The analysis was carried out according to thematic analysis based on the elements of the Chronic Care Model.

Results: The search identified 618 citations. After indepth appraisal of 327 potential citations, 62 articles that met our inclusion criteria were included in the analysis. Three IOGA main attributes were identified, which constitute IOGA's core aspects: geriatric assessment (GA), comorbidity burden and treatment outcomes. The IOGA concept comprises two broad antecedents: coordinated healthcare delivery and primary supportive care services. Regarding the consequents of an integrated approach in geriatric oncology, the studies reviewed remain inconclusive. Conclusions: Our study highlights the pioneering character of the multidimensional IOGA concept, for which the relationship between clinical and organisational attributes, on the one hand, and contextual antecedents, on the other, is not well understood. We have yet to ascertain IOGA's consequents.

Implications of key findings: There is clearly a need for a whole-system approach to change that will provide direction for multilevel (clinical, organisational, strategic) interventions to support interdisciplinary practice, education and research.

\section{ARTICLE SUMMARY}

Article focus

- An integrated oncogeriatric approach (IOGA) has emerged as a top priority within the international geriatric oncology community.

- Given the novelty of the IOGA concept and the possible contributions of the clinical and organisational domains to its development, there is a need for a common understanding of IOGA if healthcare system actors are to work together to achieve it.

- This article attempt to clarify the IOGA concept using a systematic literature review and concept analysis methods.

Key messages

- Sixty-two articles were retained for in-depth analysis.

- Over $70 \%$ of the articles were related to the development or utilisation of geriatric assessment, $50 \%$ focused on clinical guidelines to support treatment. Very few focused on care coordination or multidisciplinarity and none addressed the specific question of an integrated approach to cancer care for the elderly.

- This study highlights the pioneering character of the multidimensional IOGA concept, for which there is a lack of pertinent data to understand the nature of such a complex approach, the organisational and practice changes required, and the outcomes that may realistically be anticipated.

Strengths and limitations of this study

- The strengths of this study are the use of Rogers' concept analysis method, the integrative search strategy, the data extraction based on the Chronic Care Model and the control for bias using two researchers.

- Our focus on identifying articles that could enhance our understanding of the IOGA concept led us to include studies that were not all equally robust.

- While concepts are evolving, our study must therefore be understood as a first attempt at clarifying, at a particular point in time, an emerging concept in the field of geriatric oncology. 


\section{INTRODUCTION}

As the world's population ages, the risk of cancer increases. In developed countries, more than half of cancers occur in patients aged 70 years and older. Consequently, geriatric oncology is now at the forefront of oncology practice. Over the past 15 years, the development of clinical practices and healthcare delivery systems based on an integrated oncogeriatric approach (IOGA) has emerged as a top priority within the international geriatric oncology community. ${ }^{1}{ }^{2}$ Geriatric oncology focuses on the specific needs of elderly cancer patients related not only to their chronological age, but to the uniqueness of each person's pattern of aging. It is widely recognised that coordination and collaboration along the cancer care continuum should focus on individual needs, values and preferences, but little is said on the appropriate configurations services should adopt to achieve these goals. While evidence reports that integrated approaches are designed to overcome clinical, administrative and policy barriers and improve the quality and the security of care for elderly persons, ${ }^{3}$ there is as yet no shared definition of an integrated approach to cancer care for the elderly.

Given the complexity of the IOGA concept and the possible contributions of the clinical and organisational domains to its development, we considered that a systematic review of the literature integrating these perspectives was needed. The purpose of this study was to provide a more precise definition of an IOGA through a concept analysis.

\section{METHODS}

The IOGA concept was analysed using Rodgers' method (table 1). ${ }^{4}$ According to Rodgers, a concept is not a word, but rather the expression of the mental representations of this word at a particular point in time and in a particular context. This method entails identifying the attributes (characteristics), antecedents (preconditions for IOGA in action) and consequents (outcomes) of a concept. The concept's attributes refer to its main characteristics. Its antecedents represent the contextual factors, situations or processes that must occur beforehand and that encourage the translation of the IOGA idea into concrete practices. Finally, the consequents

\section{Table 1 Rodgers' method of concept analysis ${ }^{3}$}

1 Identify the concept of interest and associated expressions

2 Identify and select an appropriate realm (setting and sample) for data collection

3 Collect data relevant to identify: the attributes of the concept and the contextual basis of the concept

4 Analyse data regarding the above characteristics of the concept

5 Identify implications for further development of the concept pertain to the behaviours or actions resulting from the use of the concept.

\section{Search strategy}

The aim of the search strategy was to identify an appropriate realm for data collection in which to perform the IOGA concept analysis ${ }^{4}$ The strategy was based on the methodological approach to systematic and comprehensive literature reviews developed by Cooper ${ }^{5}$ and updated by Whittemore and Knafl. ${ }^{6}$ This approach can incorporate various types of studies (ie, qualitative and quantitative research) and reveal a variety of perspectives on the phenomenon of concern. We also followed the Preferred Reporting Items for Systematic Reviews and Meta-Analyses checklist as close as possible ${ }^{7}$ to the extent that it was applicable to concept analysis which is descriptive synthesis of the literature. We focused on articles published between January 2005 and April 2011. The databases searched were CINAHL, PubMed, MEDLINE, SocIndex, PsychInfo, International Political Science Abstracts, Ageline and Abstracts in Social Gerontology. Keywords used in combination were: 'elderly', '70+ years', 'older', 'old', 'cancer', 'geriatric oncology', 'geriatric oncology assessment', 'interdisciplinarity', 'multidisciplinarity', 'transdisciplinarity' 'multidisciplinary team cancer', 'multidisciplinary practice', 'interdisciplinary collaboration', 'interprofessional', 'interprofessional relations', 'collaborative care', 'health services', 'healthcare', 'healthcare services', 'healthcare reform', 'integration' and 'integrated service delivery'. The literature search was performed by two researchers (DT and KC).

\section{Article selection}

To select articles, we followed a three-phase approach. The first phase, citation selection, was based on: (1) elderly cancer patients as the study population; (2) disease management as an intervention and (3) case studies, intervention studies, assessments and evaluation studies as study characteristics. In the second phase, inclusion and exclusion criteria were gradually refined. Inclusion criteria were: (1) integration or coordination along the cancer care continuum; (2) geriatric assessment (GA) and approach; (3) cancer treatment decision-making; (4) patients aged 70 years and more and (5) multidisciplinarity. Exclusion criteria were: (1) literature reviews; (2) case reports; (3) particular therapeutic regimens; (4) opinion pieces or essays; (5) studies without any abstract; (6) professional training programmes; (7) specific episodes of the cancer care continuum (systematic screening, palliative care and cancer survivorship) and (8) access to medical insurance and treatment cost (the USA). The third phase consisted of assessing which citations (titles and abstracts) were relevant to the understanding of the concept. The initial title and abstract selection was done independently by DT and KC using the inclusion and exclusion criteria. This selection was validated by a third 
researcher, CT. Differences were resolved through discussions between DT and CT until a consensus was reached.

\section{Data analysis}

All relevant papers to be included into the full review were compiled into a formal database and managed using QDA Miner software. We developed an open-ended coding grid based on the elements of the Chronic Care Model (CCM): (1) health system-organisation of healthcare; (2) self-management support; (3) treatment decision-making support; (4) delivery system design; (5) clinical information systems and (6) community resources and policies. ${ }^{8}$ This approach enabled us to focus the analysis on concepts related to the CCM and also to integrate other concepts that emerged iteratively from our analysis. The CCM was chosen because it specifies linkages between professionals and between professionals and patients, as well as among different levels of care (eg, hospital and community care) ${ }^{89}$

The following information was systematically extracted from each article: (1) basic information on the article (title, year, authors, journal title, abstract, objectives and framework and keywords); (2) purpose of the study (evaluation tools, epidemiology, comorbidity and care coordination or system integration); (3) methods (qualitative, quantitative and mixed); (4) study design (eg, descriptive case study, randomised controlled trials and time series); (5) participants (setting, sample, data collection, procedures and tools) and (6) results. A coding process allowed us to build the thematic network of the IOGA concept's attributes, antecedents and consequents.

\section{RESULTS}

Figure 1 shows the number of citations at each of the three phases of the selection process. The search strategies identified 618 potential citations from our three broad domains. A screening of the titles and abstracts led to 327 potentially eligible citations. Based on our inclusion and exclusion criteria, 62 articles were retained for in-depth analysis. A little over $70 \%$ of the articles were related to the development or utilisation of GA tools (table 2). Half of the articles focused on clinical guidelines to support medical treatment decisionmaking and individualised care planning. Very few studies focused on care coordination or multidisciplinarity. None addressed the specific question of an integrated approach to cancer care for the elderly.

\section{Attributes}

Three main attributes were identified: GA, comorbidity and treatment outcomes. The reviewed literature on geriatric oncology frequently aimed to clarify the relationships between these attributes.

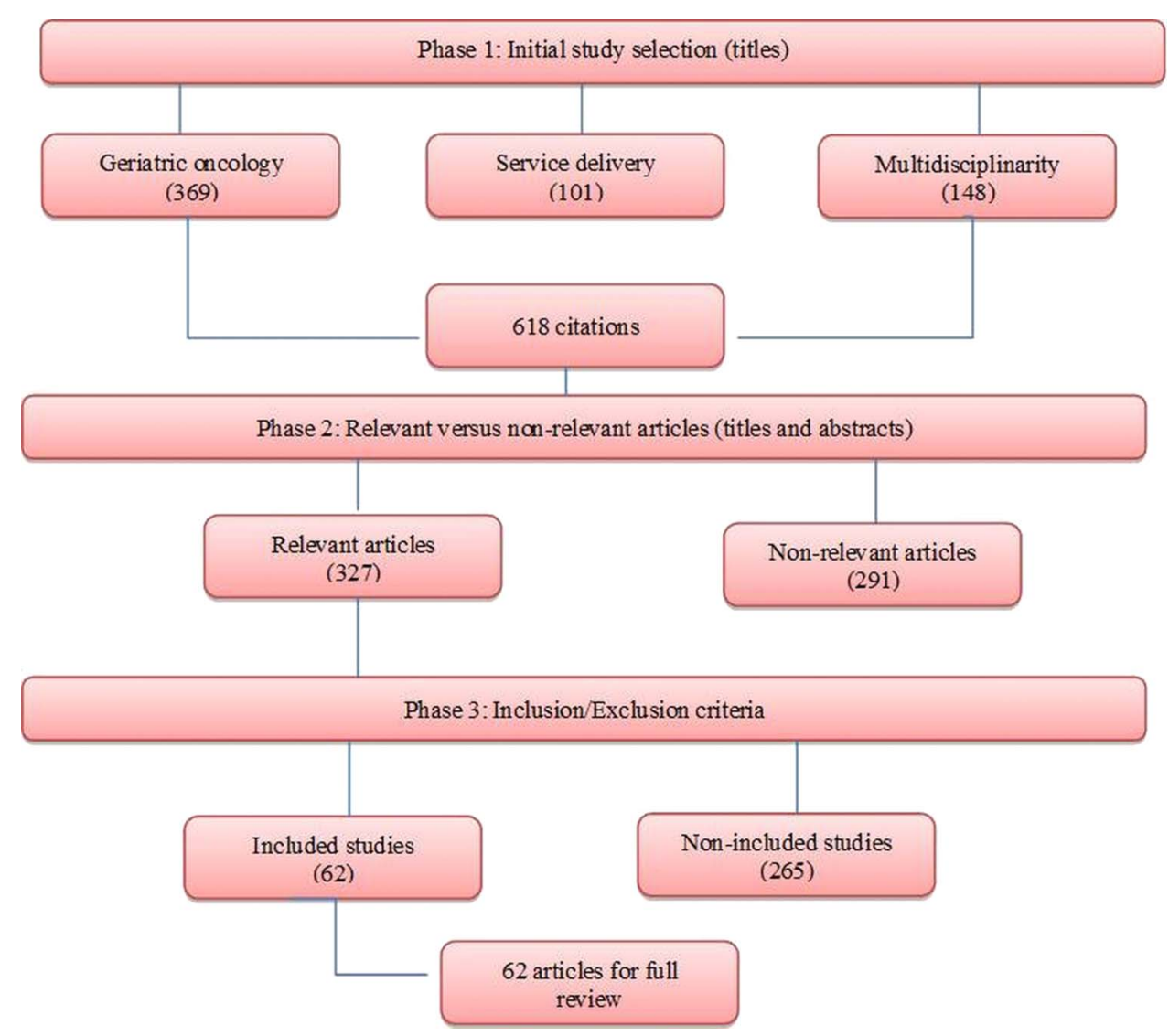

Figure 1 Article selection flow diagram. 
Table 2 Integrated oncogeriatric approach concept (attributes, antecedents and consequents)

\begin{tabular}{llll}
\hline Concepts & Results & Study references & Percentage \\
\hline Attributes & Comprehensive geriatric assessment & $10-48$ & 63 \\
& Comorbidity evaluation and management & $11-1416-1921-23252729-313537384049-68$ & 66 \\
& Treatment outcomes & $10111617262830313437384049-58$ & 45 \\
Antecedents & Coordinated healthcare delivery & 606365666970 & 8 \\
& Primary care and support services in the & 1631326971 & $181623313536566971-73$ \\
Consequents & community & & No study \\
\hline
\end{tabular}

\section{Geriatric assessment}

Development of GA tools and guidelines emerged as the cornerstone of the IOGA concept. ${ }^{10-42}$ However, the exact purpose of GA remains unclear. The consensus conference held in 1987 defined Comprehensive Geriatric Assessment (CGA) as: 'a multidisciplinary evaluation in which the multiple problems of older persons are uncovered, described and explained, if possible, and in which the resources and strengths of the person are catalogued, need for services assessed, and a coordinated care plan developed to focus interventions on the person's problems'. ${ }^{43}$ When used in geriatric oncology, CGA has been frequently cut down to the preliminary step of describing the older person's health problems. Several CGA-based tools have been developed and studied in the oncology setting. ${ }^{12-17} 19$ 22-24 26-31 373840 These tools provide data on older cancer patients' characteristics through validated geriatric tools designed to detect, but not diagnose, problems in the main dimensions of GA. ${ }^{44}$ In addition to these kinds of instruments, which can be described as multidimensional geriatric assessment tools, another group of tools has been developed recently, called screening tools, ${ }^{14} 293148$ which may, for instance, help determine which healthy older patients are candidates for standard cancer treatment.

GA has been associated with numerous benefits. It can bring to light unknown problems in domains that may clearly interfere with cancer treatment decisionmaking. ${ }^{27}{ }^{37}$ In particular, cancer treatment may generate declines in physical and functional abilities that represent crucial domains for older patients in terms of quality of life and survival. ${ }^{45}$ GA tools may help professionals ascertain a patient's functional status and then tailor cancer treatment to avoid over-treatment in patients at high risk of functional decline. ${ }^{19}$ GA has also been associated with improved tolerance to therapy and high prognostic value ${ }^{19}$ and may provide valuable prognostic factors. ${ }^{46} 47$

However, while various instruments have been studied, no definitive consensus has yet been reached regarding assessment tools' correct use and place. ${ }^{13} 14$ The current trend is to start with a screening tool to narrow the target population down to those in need of more in-depth and CGA. However, screening tools vary in their sensitivity for measuring different items. $^{13}{ }^{14}$
Consequently, depending on the choices physicians make in applying these instruments, there is a risk of over-treatment or under-treatment of patients.

Moreover, whereas the literature reviewed extols the virtues of GA, Puts et $a l^{16}$ highlight the divergences between oncologists' and geriatricians' experience of it. Oncologists usually carry out non-systematic and nonstandardised GA. Differences in perception between oncologists and geriatricians regarding the use of GA tools have also been reported by other authors. ${ }^{32}$ Hurria et $a l^{24}$ concluded there is no consensus within the geriatric oncology community regarding a standard GA instrument for older patients with cancer. International Society of Geriatric Oncology (SIOG) experts have declared they cannot recommend any specific GA tool. ${ }^{43}$ Hence, in spite of its advantages, GA is not necessarily current practice for oncologists.

\section{Comorbidity burden}

Comorbid conditions are common among ageing people, ${ }^{11-14} 16-1921-232527303537384049-66$ posing a challenge to treatment decision-making and the organisation of care. ${ }^{22}$ The comorbidity burden may thus influence integrated cancer care for elderly patients. Properly assessing the relationships between comorbid conditions and cancer treatment or patient outcomes such as functional decline and mortality is a major concern. ${ }^{11} 182540596667$ The reviewed literature provides contradictory data on key issues in geriatric oncology: (1) correlation between comorbidity and mortality and (2) comorbidity impacts on functional decline.

First, it is not clear as to whether comorbidity generates increased mortality in older patients with cancer. Alphs $e t a l,{ }^{50}$ whose aim was to predict the impact of surgical outcome on survival among elderly women with ovarian and primary peritoneal cancer, stated that comorbidity is associated with an increased risk in mortality. Janssen-Heijnen et $a l^{11}$ reported that comorbidity has an independent prognostic effect on survival among patients with colorectal and breast cancer. Another study $^{25}$ concluded that it is not so much the number of comorbid conditions that affects survival, but their severity. In fact, comorbidity was associated with survival outcomes depending on the presence of functional limitations and geriatric syndromes (delirium, falls, 
incontinence and frailty). ${ }^{68}$ In colorectal cancer patients, comorbidity negatively influenced cancerspecific mortality, and not overall survival, whereas geriatric syndromes and functional limitations affected overall survival. ${ }^{74}$ Arnoldi $e t a l^{88}$ made a similar observation regarding an outpatient population. Considering these results, the presence of comorbidity does not appear to have a direct prognostic effect on mortality, and its severity matters more than the fact of its absence or presence. Therefore, the extent to which comorbidity has an independent prognostic effect on mortality is challenged by certain studies reporting that the effect of comorbidity on survival is contingent upon patients' functional status and the severity of their comorbid conditions.

Second, comorbidity's impact on functional decline is also controversial. Gironés et $a l^{22}$ reported that comorbid conditions had little impact on functional decline among elderly breast cancer patients. However, it remains difficult to ascertain whether it is the aging process or the cancer treatment that produces functional decline. Although Kurtz et $a l^{66}$ underlined the role of functional decline in increased hospital admission rates, they failed to determine the role of the cancer itself, as opposed to other comorbid conditions, in the decrease of physical functioning.

Relationships between comorbidity and functional decline have therefore not been clearly demonstrated in geriatric oncology. In some studies, comorbidity affected patient outcomes. ${ }^{11} 3850$ In others, functional decline worsened following cancer treatment ${ }^{17} 31$ and affected survival when combined with comorbidity. However, studies focused on this issue reported no association between comorbidity and functional decline, which speaks to the lack of validated measurement methods rather than to the real absence of relationships ${ }^{67}$ The development of such instruments may not only help clarify the interactions between comorbidity and functional decline, but also their potential impact on survival.

\section{Treatment outcomes}

Regarding treatment outcomes, ${ }^{10} 11161726283031343740$ 49-58 $60 \quad 63 \quad 65 \quad 66 \quad 69$ the main preoccupation in geriatric oncology revolves around finding the most effective treatment regimen with an acceptable toxicity risk. While ultimately the goal remains cancer control, greater consideration must be given to the variability of elderly cancer patients' health status and independence. On the one hand, patients may be undertreated because of physicians' fears regarding their patients' greater susceptibility to toxicity. On the other hand, standard protocols may lead to increased risk of toxicity and overtreatment for some patients. ${ }^{70}$ Moreover, cancer stage may sometimes be underestimated if patients' nonspecific symptoms are attributed to comorbid conditions or the ageing process. ${ }^{10}$ Optimal functional status represents a critical outcome in older cancer patients. The association between cancer treatment and functional decline remains an open question. More specifically, fatigue brought on by cancer treatment may accelerate functional decline. ${ }^{17}$ Functional dependence was more frequently observed in patients who had surgery or radiation therapy in addition to chemotherapy. ${ }^{31}$ Impairment may affect instrumental activities of daily living, in particular the capacity to drive and/or use public transportation. ${ }^{22}$ However, in older cancer outpatients, functional status was reasonably preserved, even among patients considered frail. ${ }^{38}$ Similar proportions of patients with functional decline were observed between cancer and non-cancer older patients admitted to a geriatric unit. ${ }^{60}$ The relationships between cancer treatment and functional decline thus remain unclear because of the heterogeneity of patients' characteristics in the studies reviewed, especially cancer type, stage and treatment, functional status measurement methods and time of the functional assessment.

Some attention was given to the impact of various support services on patient outcomes. On the one hand, GA may help define older cancer patients' health status, especially risk factors for frailty; GA may also clarify patients' needs for specific services. However, patient outcomes also depend on cancer characteristics, which explain why survival might not be quite different in patients with advanced disease receiving cancer treatment or supportive care. ${ }^{38}$ On the other hand, a study conducted in an acute care geriatric ward found that older cancer patients received suboptimal cancer treatment and were more likely to suffer from depression and malnutrition. ${ }^{57}$ The need for a more complete assessment to detect geriatric problems in older cancer patients admitted to geriatric wards was underlined by the same authors. Finally, the review emphasised the need for a more responsive healthcare system due to the intricacy of older patients' health problems. ${ }^{69}$

\section{Antecedents}

The reviewed literature showed that the IOGA concept comprises two broad antecedents: coordinated healthcare delivery and primary care and support services in

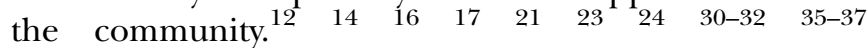
$394956 \quad 6162 \quad 6469 \quad 71-73 \quad 75$

\section{Coordinated healthcare delivery}

Coordinated healthcare delivery refers to the patterns of interaction between healthcare professionals within an interdisciplinary team in order to successfully meet the needs of patients and, in particular, ensure that health and social services are delivered in tandem and according to a patient's specific needs. ${ }^{16} 32697176$ In the literature on geriatric oncology, multidisciplinary teamwork is considered the core mechanism to improve both collaboration and care coordination. Emphasis is put on the relationships between oncologists and geriatricians. In one study that examined oncologists' and geriatricians' views of the French geriatric oncology system, which 
aims to improve care coordination for elderly cancer patients, the authors observed multiple heterogeneous practices based on local resources and care models that left geriatricians' involvement in patient with cancer care, most often, incomplete. ${ }^{32}$ According to the authors, the successful implementation of an integrated approach is challenged by everyday work relationships as well as by professional turf protection. A Canadian study ${ }^{16}$ explored the relationship between oncologists and geriatricians within a university hospital. The authors recommended more effective collaboration between specialists. Hence, coordination of care is crucially defined by interprofessional relationships between geriatric and oncology teams.

There are also calls for improvements at the organisational level, although they are nascent. For example, Puts $e a^{16}$ recommended the creation of networks that would include geriatricians. More broadly, there is a call in the geriatric oncology literature to organise the healthcare system so that the needs of the elderly cancer population, and particularly functional outcomes, will be properly taken into consideration. ${ }^{31}$

\section{Primary care and support services in the community}

Numerous services are considered necessary for appropriate care of elderly cancer patients. In particular, access to social services, mental health services, transportation services and home care services would help limit patient hospitalisations motivated by social reasons. ${ }^{12} 1623 \quad 353656 \quad 6971-73$ The association between lack of social support and depression was raised in one study. ${ }^{23}$ Furthermore, elderly patients living alone tended not only to be depressed, but also to be malnourished, which may cause poor tolerance to cancer treatment. ${ }^{12}$ Also, living alone, not driving, mobility and vision problems and a willingness to maintain independence were reported to affect the cancer care experience. ${ }^{71}$ Elderly cancer patients seemed reluctant to assert their needs to healthcare professionals, thereby decreasing the probability of those needs being met. ${ }^{71}$ This situation led Kurtz et $a l^{35}$ to refer to 'unmet needs' as 'unknown needs'. Kahana $e t a l^{73}$ reported that elderly patients may remain cautious and passive about seeking social support. However, a transition in older patients' behaviour has been observed from passivity to proactive consumerism, suggesting the possibility of fruitful healthcare partnership between patients and professionals. ${ }^{73}$ Providing the appropriate social support for elderly cancer patients thus depends on the extent to which the latter are given the opportunity to voice their needs.

In terms of primary care, the reviewed literature indicated variability regarding referrals of elderly cancer patients. While referral rates decreased as a function of age, ${ }^{12} 35$ age itself was not the overriding factor for the lower frequency of referrals. ${ }^{35}$ Functional dependency, ${ }^{31}{ }^{35}$ comorbidity and patients' preferences ${ }^{35}$ were also important parameters. For instance, Goodwin $e t a l^{81}$ reported that functional dependency may influence clinicians' care plans, as well as patients' adherence to treatment. This tended to result in undertreatment of cancer in elderly patients. Finally, shortcomings in multidisciplinary teamwork may also translate into less frequent referrals. In particular, Lynch $e t a \rho^{6}$ reported that patients seen by a social worker were not referred for evaluation into the programme, whereas those seen by a nurse or a physician were referred.

A few other studies focused more squarely on the role of families and/or caregivers. ${ }^{56}{ }^{72}$ While there are calls for the family to take part in treatment decisionmaking, ${ }^{56}$ such help may be at times 'neutrally received' by the elderly patient. ${ }^{71}$ Since a patient's perception of the availability of social support services may play a role in treatment refusal, Sinding $e t a l^{71}$ stressed the need for 'adequately resourced and skilled hospital care and sufficient, consistent and reliable community care', particularly because of some patients' reluctance to accept help from family members. While families and caregivers play an important role in terms of social support, elderly patients' reluctance to seek help requires that skilled primary healthcare professionals accompany caregivers.

\section{Consequents}

Regarding the consequents of IOGA, the studies reviewed were inconclusive. No study specifically explored how different organisational models of care may shape the cancer care experience for the elderly. Cancer treatment outcomes have been examined more closely than the overall cancer care experience.

In sum, the IOGA concept may be usefully defined as follows: IOGA refers to a coherent and coordinated set of services that are planned, managed and delivered to elderly cancer patients across a range of acute and primary care settings and by a range of collaborating care providers including oncologists, geriatricians, interprofessional team members and lay caregivers. The essence of IOGA is that elderly cancer patients and their loved ones receive whatever best-practice-based services they need, when and where they need them, in order to optimise health status and independence, and that all services are delivered from a whole-system perspective.

\section{DISCUSSION}

To date, IOGA mostly revolves around four main attributes, that is, CGA, functional decline, comorbidity evaluation and management, and treatment outcomes. More specifically, one challenge of the IOGA concept is to assess the relationships between cancer, comorbidity, functional decline and patient outcomes. While there is still uncertainty in the literature on the nature of these relationships, there is also great variability in the measurement methods used to evaluate functional decline as well as comorbidity. In fact, there is no consensus on a recommended assessment tool. The literature suggested actual interactions between comorbidity, functional decline and GA, but these were not supported by robust 
evidence. This can be attributed to the variability in research designs and in the instruments used to measure comorbidity and functional decline, as well as to the relatively recent emergence of the concept of geriatric oncology. Moreover, to date, geriatric oncology and an integrated approach to cancer care are two separate worlds, where coordination and integration mechanisms depend in part on patients' capacity to activate them. As shown in the figure we developed on the basis of our literature review, IOGA is still mired in a two-discipline geriatric and oncology dilemma (figure 2). ${ }^{77}$

Beyond key issues like GA, functional decline and comorbidity, the complexity of cancer care among the elderly population justifies the need to focus attention and research on other aspects as well. Studies examining social support indicate that the lack of integration between health and social services complicates older patients' cancer care experience, causing reduced tolerance to cancer treatment. The literature focused on social support systems may thus enhance the accuracy of knowledge on the complex nature of the disease by underlining its social dimension, as well as evaluating the anticipated quality, security and efficiency outcomes. Our resulting definition of IOGA espouses most of the elements of the CGM developed by Barr et al The CCM focuses on chronic disease management and has been used to develop a model for cancer care quality. ${ }^{78}$ However, while the CCM specifically supports multidisciplinary care, patient-professional relationships and outcomes, decision-makers also need strong scientific evidence upon which to base healthcare policies and programmes. Nevertheless, the CCM adequately supported our purpose of defining the IOGA concept, particularly with regard to: (1) self-management and decision support; (2) delivery system design and (3) healthcare policy.

Self-management and decision support refer to 'a person's ability to manage the symptoms and the consequences of living with a chronic condition, including treatment, physical, social and lifestyle changes' ${ }^{79}$ Self-management involves productive interactions between the patient and a multidisciplinary team to develop a collaborative care plan. Such interactions also enable decision support. Multidisciplinary teamwork ensures the coordination of patient-centred care and patients' active role in their own care plan. However, in the literature, data on individualised management are limited to care attributes, toxicity grading and management of polypharmacy. The cancer treatment decisionmaking process remains limited to treatment options, although the role of patients, families and caregivers is acknowledged. Geriatric oncology teams must in the future encourage older patients to become proactive and feel involved in their own care plan and use of healthcare services. IOGA also aims to facilitate multidisciplinary coordination of biomedical and psychosocial care. ${ }^{78}$ Delivering an integrated cancer care system requires developing efficient networks between hospitals, primary healthcare facilities, human and social services and communities. ${ }^{76}$ Formalising such networks facilitates the seamless delivery of services ${ }^{76}$ and the comprehensive assessment of the patient's multiple evolving needs. Integrated cancer care in geriatric oncology revolves mainly around multidisciplinary teamwork and the use of GA. However, as previously emphasised, there is a significant lack of care coordination and of consensus on GA instruments. In terms of multidisciplinary teamwork, attention is focused mainly on the relationships between oncologists and geriatricians and less so on other professionals, reflecting a lack of recognition of the key position played by some professionals in accompanying patients on their cancer care pathway. Such attention may also reinforce professional silos, to the detriment of a whole-system approach. In geriatric oncology, GA has mainly been considered as an instrument to facilitate the cancer treatment decision-making process for the individual patient. The role of GA in care coordination for geriatric cancer patients remains
Figure 2 Integrated Oncogeriatric Approach (IOGA) Model

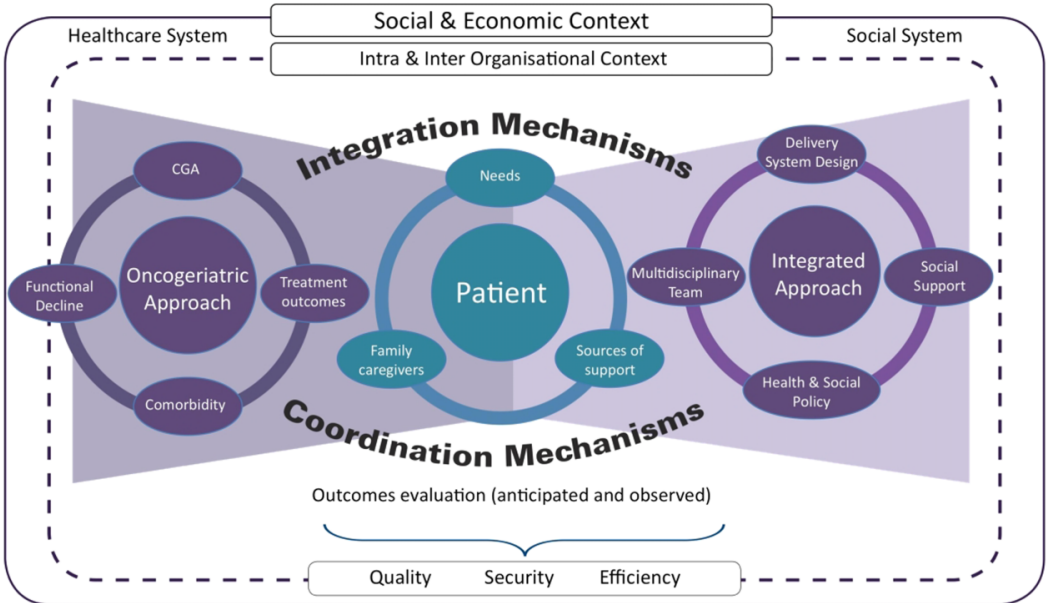


underevaluated, in comparison with the geriatric noncancer population.

An integrated model of cancer care also requires innovations at the policy level regarding three key components: (1) delivery system design; (2) clinical information systems and (3) multidisciplinary care. Decision-makers develop policies composed of norms, incentives and regulations intended to facilitate the implementation of these three components and avoid the classic trap of reproducing new professional silos. The decision-making process around the development of such policies is also important. The effectiveness of such policies depends on the extent to which stakeholders, and in particular healthcare professionals and patients, are included in the policy process. This ensures that the norms, incentives and regulations are evidence based. Including healthcare professionals and patients in the policy process requires establishing governance mechanisms that engage various healthcare stakeholders in the development of policies around integrated cancer care. Apart from calls for changes at the policy level, the geriatric oncology literature has not identified the specific changes required to foster integrated cancer care, nor has it properly defined the role of healthcare professionals and patients in the policy-making process.

\section{Strengths and limitations of the study}

Our study highlights the pioneering character of the multidimensional IOGA concept, for which the relationship between clinical and organisational attributes, on the one hand, and contextual antecedents, on the other, is not well understood. However, there are some weaknesses. While the CCM is widely recognised in the field of chronic disease management, in using this general model we may have left out other concepts that could contribute significantly to refining the IOGA concept. Also, because our literature search covered three broad domains (geriatric oncology, integrated service delivery and multidisciplinarity), certain relevant articles may have been missed in spite of our systematic approach. Indeed, this limitation was reflected in the keywords chosen, such that, for example, the term 'comorbidity' was not included in the search strategy, and consequently, the number of articles focusing on this important clinical aspect may have been under-represented in our sample. It may also be that our focus on identifying articles that could enhance our understanding of the IOGA concept led us to include studies that were not all equally robust. Finally, as stated by Rodgers ${ }^{80}$, while concepts are evolving, concept analysis can only provide a snapshot of a given concept. Our study must therefore be understood as a first attempt at clarifying, at a particular point in time, an emerging concept in the field of geriatric oncology.

\section{Implications for clinicians and policymakers}

By highlighting ways to envision IOGA conceptually, our analysis of its attributes, antecedents and consequents represents an attempt to provide guidance to clinicians and decision-makers in the design of improved healthcare delivery models. Our in-depth analysis of the IOGA concept underscores the lack of pertinent data for really understanding the nature of such a complex approach, the organisational and practice changes required, and the outcomes that may realistically be anticipated for older patients with cancer, as well as for professional and lay care providers. The scarcity of conceptual and empirical evidence focusing on IOGA may reflect researchers' predominant interest in cancer treatment options adapted for older adults' specific conditions. In this context, the result of our integrative review is disappointing, since it does not provide solid ground to clarify the IOGA concept. Nevertheless, our study contributes to worldwide efforts to improve care at the interface of cancer and ageing. It may constitute a starting point for promoters of comprehensive cancer care, since they will need a common understanding of IOGA if they are to work together to achieve it.

\section{Author affiliations}

${ }^{1}$ Centre de recherche CSSS Champlain-Charles-Le Moyne, Université de Sherbrooke, École des Sciences infirmières, Longueuil, Québec, Canada ${ }^{2}$ Centre de recherche CSSS Champlain-Charles Le Moyne, Longueuil,Québec, Canada

${ }^{3}$ Programme d'oncologie gériatrie, Département d'oncologie, Centre LeonBérard, Claude-Bernard Lyon-1 Université Lyon, Lyon, France

${ }^{4}$ Centre de recherche CSSS Champlain-Charles-Le Moyne, Université de Sherbrooke, Longueuil, Québec, Canada

${ }^{5}$ Centre intégré de cancérologie de la Montérégie, Greenfield Park, Québec, Canada, Université de Sherbrooke, Faculté de médecine et des sciences de la santé, Longueuil. Québec, Canada

Contributors DT and KC led on conception design, conducted the literature search, interpreted and analysed the data, as well as wrote the manuscript. CT, SJ and JL contributed to the design and critically appraised the content of the manuscript. All authors approved the final version of the article submitted.

Funding This research received no specific grant from any funding agency in the public, commercial or not-for-profit sectors.

Competing interests None.

Provenance and peer review Not commissioned; externally peer reviewed.

Data sharing statement No additional data are available.

\section{REFERENCES}

1. Société internationale d'oncologie gériatrique/International Society of Geriatric Oncology (SIOG). The SIOG 10 Priorities Initiative. Société internationale d'oncologie gériatrique/International Society of Geriatric Oncology 2011. http://www.siog.org/images/SIOG_ documents/siog_10_priorities_final.pdf(accessed 24 Jan 2012).

2. Extermann M, Aapro M, Audisio R, et al. Main priorities for the development of geriatric oncology: a worldwide expert perspective. J Geriatr Oncol 2011;2:270-3.

3. Béland F, Hollander MJ. Integrated models of care delivery for the frail elderly: international perspectives. Gac Sanit 2011;25(Suppl 2):138-46.

4. Rodgers BL, Knalf KA. Concept developement in nursing: foundations, techniques and applications. Philadelphia: W.B. Saunders, 2000.

5. Cooper $\mathrm{H}$. The integrative research review: a systematic approach. Beverley Hills, CA: Sage, 1984.

6. Whittemore R, Knafl K. The integrative review: updated methodology. J Adv Nurs 2005;52:546-53.

7. Liberati A, Altman DG, Tetzlaff J, et al. The PRISMA statement for reporting systematic reviews and meta-analyses of studies that 
evaluate healthcare interventions: explanation and elaboration. $B M J$ 2009;339:b2700.

8. Barr VJ, Robinson S, Marin-Link B, et al. The expanded Chronic Care Model: an integration of concepts and strategies from population health promotion and the Chronic Care Model. Hosp Q 2003;7:73-82.

9. Katz SJ, Hawley ST, Morrow M, et al. Coordinating cancer care: patient and practice management processes among surgeons who treat breast cancer. Med Care 2010;48:45-51.

10. Freyer G, Braud AC, Chaibi P, et al. Dealing with metastatic breast cancer in elderly women: results from a French study on a large cohort carried out by the 'Observatory on Elderly Patients'. Ann Oncol 2006;17:211-16.

11. Janssen-Heijnen ML, Maas HA, Houterman S, et al. Comorbidity in older surgical cancer patients: influence on patient care and outcome. Eur J Cancer 2007;43:2179-93.

12. Marenco D, Marinello $R$, Berruti A, et al. Multidimensional geriatric assessment in treatment decision in elderly cancer patients: 6-year experience in an outpatient geriatric oncology service. Crit Rev Oncol Hematol 2008;68:157-64.

13. Luciani A, Ascione G, Bertuzzi C, et al. Detecting disabilities in older patients with cancer: comparison between comprehensive geriatric assessment and vulnerable elders survey-13. J Clin Oncol 2010;28:2046-50.

14. Kellen E, Bulens $P$, Deckx L, et al. Identifying an accurate pre-screening tool in geriatric oncology. Crit Rev Oncol Hematol 2010;75:243-8.

15. Retornaz F, Monette J, Batist G, et al. Usefulness of frailty markers in the assessment of the health and functional status of older cancer patients referred for chemotherapy: a pilot study. J Gerontol A Biol Sci Med Sci 2008;63:518-22.

16. Puts MT, Girre V, Monette J, et al. Clinical experience of cancer specialists and geriatricians involved in cancer care of older patients: a qualitative study. Crit Rev Oncol Hematol 2010;74:87-96.

17. Luciani $A$, Jacobsen PB, Extermann $M$, et al. Fatigue and functional dependence in older cancer patients. Am J Clin Oncol 2008;31: 424-30.

18. Koroukian SM, Murray P, Madigan E. Comorbidity, disability, and geriatric syndromes in elderly cancer patients receiving home health care. J Clin Oncol 2006;24:2304-10.

19. Cudennec T, Gendry T, Labrune S, et al. Use of a simplified geriatric evaluation in thoracic oncology. Lung Cancer 2010;67:232-6.

20. Terret C, Perol D, Albrand G, et al. Quality of life in geriatric oncologyan evaluation of standard questionnaires in elderly men with urological malignancies. Crit Rev Oncol Hematol 2011;77:201-9.

21. Monfardini S, Aapro MS, Bennett JM, et al. Organization of the clinical activity of geriatric oncology: report of a SIOG (International Society of Geriatric Oncology) task force. Crit Rev Oncol Hematol 2007;62:62-73.

22. Gironés R, Torregrosa D, Dìaz-Beveridge R. Comorbidity, disability and geriatric syndromes in elderly breast cancer survivors. Results of a single-center experience. Crit Rev Oncol Hematol 2010;73:236-45.

23. Molina-Garrido MJ, Guillen-Ponce C. Development of a cancer-specific Comprehensive Geriatric Assessment in a University Hospital in Spain. Crit Rev Oncol Hematol 2011;77:148-61.

24. Hurria A, Gupta S, Zauderer M, et al. Developing a cancer-specific geriatric assessment: a feasibility study. Cancer 2005;104:1998-2005.

25. Koroukian SM. Assessment and interpretation of comorbidity burden in older adults with cancer. J Am Geriatr Soc 2009;57(Suppl 2): S275-8.

26. Massa E, Madeddu C, Astara G, et al. An attempt to correlate a 'Multidimensional Geriatric Assessment' (MGA), treatment assignment and clinical outcome in elderly cancer patients: results of a phase II open study. Crit Rev Oncol Hematol 2008;66:75-83.

27. Girre V, Falcou MC, Gisselbrecht M, et al. Does a geriatric oncology consultation modify the cancer treatment plan for elderly patients? $J$ Gerontol A Biol Sci Med Sci 2008;63:724-30.

28. Minisini AM, De Faccio S, Ermacora $P$, et al. Cognitive functions and elderly cancer patients receiving anticancer treatment: a prospective study. Crit Rev Oncol Hematol 2008:67:71-9.

29. Mohile SG, Bylow K, Dale W, et al. A pilot study of the vulnerable elders survey-13 compared with the comprehensive geriatric assessment for identifying disability in older patients with prostate cancer who receive androgen ablation. Cancer 2007;109:802-10.

30. Wedding U, Kodding D, Pientka L, et al. Physicians' judgement and comprehensive geriatric assessment (CGA) select different patients as fit for chemotherapy. Crit Rev Oncol Hematol 2007;64:1-9.

31. Goodwin JA, Coleman EA, Shaw J. Short Functional Dependence Scale: development and pilot test in older adults with cancer. Cancer Nurs 2006;29:73-81
32. Sifer-Riviere L, Girre V, Gisselbrecht M, et al. Physicians' perceptions of cancer care for elderly patients: a qualitative sociological study based on a pilot geriatric oncology program. Crit Rev Oncol Hematol 2010;75:58-69.

33. Snyder DC, Morey MC, Sloane R, et al. Reach out to ENhancE Wellness in Older Cancer Survivors (RENEW): design, methods and recruitment challenges of a home-based exercise and diet intervention to improve physical function among long-term survivors of breast, prostate, and colorectal cancer. Psychooncology 2009;18:429-39.

34. McBean AM, Yu X, Virnig BA. The use of preventive health services among elderly uterine cancer survivors. Am J Obstet Gynecol 2008;198:86.e1-8.e1.

35. Kurtz JE, Heitz D, Enderlin P, et al. Geriatric oncology, general practitioners and specialists: current opinions and unmet needs. Crit Rev Oncol Hematol 2010;75:47-57.

36. Lynch MP, Marcone D, Kagan SH. Developing a multidisciplinary geriatric oncology program in a community cancer center. Clin $J$ Oncol Nurs 2007;11:929-33.

37. Aparicio T, Girard L, Bouarioua N, et al. A mini geriatric assessment helps treatment decision in elderly patients with digestive cancer. A pilot study. Crit Rev Oncol Hematol 2011;77:63-9.

38. Arnoldi E, Dieli M, Mangia M, et al. Comprehensive geriatric assessment in elderly cancer patients: an experience in an outpatient population. Tumori 2007;93:23-5

39. Sifer-Riviere L, Saint-Jean O, Gisselbrecht M, et al. What the specific tools of geriatrics and oncology can tell us about the role and status of geriatricians in a pilot geriatric oncology program. Ann Oncol 2011;22:2325-29.

40. Wedding U, Rohrig B, Klippstein A, et al. Age, severe comorbidity and functional impairment independently contribute to poor survival in cancer patients. J Cancer Res Clin Oncol 2007;133:945-50.

41. Wieland D, Hirth V. Comprehensive geriatric assessment. Cancer Control 2003;10:454-62.

42. Conroy SP, Stevens T, Parker SG, et al. A systematic review of comprehensive geriatric assessment to improve outcomes for frail older people being rapidly discharged from acute hospital: 'interface geriatrics'. Age Ageing 2011;40:436-43.

43. Extermann M, Aapro M, Bernabei R, et al. Use of comprehensive geriatric assessment in older cancer patients: recommendations from the task force on CGA of the International Society of Geriatric Oncology (SIOG). Crit Rev Oncol Hematol 2005;55:241-52.

44. Terret C. How and why to perform a geriatric assessment in clinical practice. Ann Oncol 2008;19(Suppl 7):vii300-3.

45. Audisio RA, Pope D, Ramesh HS, et al. Shall we operate? Preoperative assessment in elderly cancer patients (PACE) can help. A SIOG surgical task force prospective study. Crit Rev Oncol Hematol 2008;65:156-63.

46. Fukuse T, Satoda N, Hijiya K, et al. Importance of a comprehensive geriatric assessment in prediction of complications following thoracic surgery in elderly patients. Chest 2005;127:886-91.

47. Kristjansson SR, Nesbakken A, Jordhoy MS, et al. Comprehensive geriatric assessment can predict complications in elderly patients after elective surgery for colorectal cancer: a prospective observational cohort study. Crit Rev Oncol Hematol 2010;76: 208-17.

48. Terret C, Albrand G, Moncenix G, et al. Karnofsky Performance Scale (KPS) or Physical Performance Test (PPT)? That is the question. Crit Rev Oncol Hematol 2011;77:142-7.

49. Rao AV, Hsieh F, Feussner JR, et al. Geriatric evaluation and management units in the care of the frail elderly cancer patient $J$ Gerontol A Biol Sci Med Sci 2005;60:798-803.

50. Alphs $\mathrm{HH}$, Zahurak ML, Bristow RE, et al. Predictors of surgical outcome and survival among elderly women diagnosed with ovarian and primary peritoneal cancer. Gynecol Oncol 2006;103:1048-53.

51. Hardiman KM, Cone M, Sheppard BC, et al. Disparities in the treatment of colon cancer in octogenarians. Am J Surg 2009;197:624-8.

52. Rutten HJ, den Dulk M, Lemmens VE, et al. Controversies of total mesorectal excision for rectal cancer in elderly patients. Lancet Oncol 2008;9:494-501.

53. Iwamoto FM, Reiner AS, Panageas KS, et al. Patterns of care in elderly glioblastoma patients. Ann Neurol 2008;64:628-34.

54. Nurgalieva Z, Xia R, Liu CC, et al. Risk of chemotherapy-induced peripheral neuropathy in large population-based cohorts of elderly patients with breast, ovarian, and lung cancer. Am J Ther 2010;17:148-58.

55. Lane BR, Abouassaly R, Gao T, et al. Active treatment of localized renal tumors may not impact overall survival in patients aged 75 years or older. Cancer 2010;116:3119-26. 
56. Kanaskie ML, Tringali CA. Promoting quality of life for geriatric oncology patients in acute care and critical care settings. Crit Care Nurs Q 2008;31:2-11.

57. Retornaz F, Seux V, Pauly V, et al. Geriatric assessment and care for older cancer inpatients admitted in acute care for elders unit. Crit Rev Oncol Hematol 2008;68:165-71.

58. Mancuso A, Migliorino M, De Santis S, et al. Correlation between anemia and functional/cognitive capacity in elderly lung cancer patients treated with chemotherapy. Ann Oncol 2006;17:146-50.

59. Wedding U, Roehrig B, Klippstein A, et al. Comorbidity in patients with cancer: prevalence and severity measured by cumulative illness rating scale. Crit Rev Oncol Hematol 2007;61:269-76.

60. Retornaz F, Seux V, Sourial N, et al. Comparison of the health and functional status between older inpatients with and without cancer admitted to a geriatric/internal medicine unit. J Gerontol A Biol Sci Med Sci 2007;62:917-22.

61. Townsley CA, Chan KK, Pond GR, et al. Understanding the attitudes of the elderly towards enrolment into cancer clinical trials. BMC Cancer 2006;6:34.

62. Schootman M, Jeffe DB, Lian M, et al. Area-level poverty is associated with greater risk of ambulatory-care-sensitive hospitalizations in older breast cancer survivors. J Am Geriatr Soc 2008;56:2180-7.

63. Kurtz ME, Kurtz JC, Given CW, et al. Predictors of use of health care services among elderly lung cancer patients: the first year after diagnosis. Support Care Cancer 2006;14:243-50.

64. Punglia RS, Weeks JC, Neville BA, et al. Effect of distance to radiation treatment facility on use of radiation therapy after mastectomy in elderly women. Int $J$ Radiat Oncol Biol Phys 2006;66:56-63.

65. Locher JL, Kilgore ML, Morrisey MA, et al. Patterns and predictors of home health and hospice use by older adults with cancer. J Am Geriatr Soc 2006;54:1206-11.

66. Kurtz ME, Kurtz JC, Given CW, et al. Utilization of services among elderly cancer patients-relationship to age, symptoms, physical functioning, comorbidity, and survival status. Ethn Dis 2005;15 S17-22.

67. Groll DL, To T, Bombardier C, et al. The development of a comorbidity index with physical function as the outcome. J Clin Epidemiol 2005;58:595-602.
68. Inouye SK, Studenski S, Tinetti ME, et al. Geriatric syndromes: clinical, research, and policy implications of a core geriatric concept. J Am Geriatr Soc 2007;55:780-91.

69. Molleman E, Broekhuis M, Stoffels R, et al. How health care complexity leads to cooperation and affects the autonomy of health care professionals. Health Care Anal 2008;16:329-41.

70. Basso U, Tonti S, Bassi C, et al. Management of Frail and Not-Frail elderly cancer patients in a hospital-based geriatric oncology program. Crit Rev Oncol Hematol 2008;66:163-70.

71. Sinding C, Wiernikowski J, Aronson J. Cancer care from the perspectives of older women. Oncol Nurs Forum 2005;32:1169-75.

72. Grov EK, Eklund ML. Reactions of primary caregivers of frail older people and people with cancer in the palliative phase living at home. $J$ Adv Nurs 2008;63:576-85.

73. Kahana E, Kahana B, Kelley-Moore J, et al. Toward advocacy in cancer care for older adults: survivors have cautious persona actions but bold advice for others. J Am Geriatr Soc 2009;57 S269-71.

74. Koroukian SM, Xu F, Bakaki PM, et al. Comorbidities, functional limitations, and geriatric syndromes in relation to treatment and survival patterns among elders with colorectal cancer. $J$ Gerontol $A$ Biol Sci Med Sci 2010;65:322-9.

75. Baldwin LM, Cai $Y$, Larson EH, et al. Access to cancer services for rural colorectal cancer patients. J Rural Health 2008;24:390-9.

76. Kodner DL. All together now: a conceptual exploration of integrated care. Healthc Q 2009;13:6-15.

77. Tremblay D, Charlebois K, Joannette S, et al. Towards an integrated oncogeriatric approach: looking beyond the two discipline dilemma. $J$ Geriatr Oncol 2011;2 S25.

78. Wagner E. Models for patient-centered cancer care. Washington DC Cancer Research Network; 2011. http://iom.edu/ /media/Files/ Activity\%20Files/Disease/NCPF/2011-Feb-28-Treatment-Planning/ Wagner\%20Presentation\%20for\%20WEB.pdf(accessed 24 Jan 2012).

79. McCorkle R, Ercolano E, Lazenby M, et al. Self-management: enabling and empowering patients living with cancer as a chronic illness. CA Cancer J Clin 2011;61:50-62.

80. Rodgers B. Concept analysis: an evolutionary view. In: Rodgers B, Knafl KA, eds. Concept development in nursing, 2nd edn. Philadelphia: W. B. Saunders, 2000:77-102. 